\title{
Use of Hydrofluoric Acid as Mineralizer in Hydrothermal and Organothermal Synthesis of $\mathrm{Me}^{2+}$-Substituted Aluminophosphates. I
}

\author{
A. Nørlund Christensen* and R. G. Hazell \\ Department of Inorganic Chemistry, Aarhus University, DK-8000 Aarhus C, Denmark
}

\begin{abstract}
Christensen, A. N. and Hazell, R. G., 1999. Use of Hydrofluoric Acid as Mineralizer in Hydrothermal and Organothermal Synthesis of $\mathrm{Me}^{2+}$-Substituted Aluminophosphates. I. - Acta Chem. Scand. 53: 403-409. (C) Acta Chemica Scandinavica 1999.

Hydrofluoric acid has been used as mineralizer in hydrothermal and organothermal syntheses of $\mathrm{Me}^{2+}$-substituted aluminophosphates at temperatures up to $180^{\circ} \mathrm{C}$. As structure-directing templates triethylamine, di- $n$-propylamine, tripropylamine, 1,4-diazabicyclo[2.2.2] octane and ethylenediamine were used. Single crystals large enough for traditional X-ray single crystal diffraction analyses were obtained of $\mathrm{ZnAPO}-5$, AFI, $(\mathrm{Zn}, \mathrm{Al}) \mathrm{PO}_{4} \cdot 0.5 \mathrm{C}_{2} \mathrm{H}_{10} \mathrm{~N}_{2}$, CoAPO5, AFI, $\mathrm{CoAl}\left(\mathrm{PO}_{4}\right)_{2} \cdot \mathrm{C}_{2} \mathrm{H}_{9} \mathrm{~N}_{2},(\mathrm{Co}, \mathrm{Al}) \mathrm{PO}_{4} \cdot 0.5 \mathrm{C}_{2} \mathrm{H}_{10} \mathrm{~N}_{2}$ and CoAPO-43, GIS. The microporous compound ZnAPO-35, LEV, was obtained as a powder. The compounds were identified from X-ray powder diffraction patterns and from single crystal $\mathrm{X}$-ray diffraction analysis. The crystal structure of $\left(\mathrm{Co}_{0.84} \mathrm{Al}_{0.16}\right) \mathrm{PO}_{4} \cdot 0.5 \mathrm{C}_{2} \mathrm{H}_{10} \mathrm{~N}_{2} \cdot 0.5 \mathrm{H}_{2} \mathrm{O}$ is reported. The unit cell dimensions are $a=10.1724(6)$ and $c=9.6060(6) \AA$, and model calculations were made using the space group $I \overline{4} 2 \mathrm{~m}$.
\end{abstract}

Heterogeneous mixtures of solids and water are heated under pressure at temperatures over $100^{\circ} \mathrm{C}$ in hydrothermal reactions. This can result in compound formation by chemical reactions or in recrystallization of solids to produce crystalline powders or single crystals. Such reactions performed in pressure vessels can also be made with an organic solvent as the reaction medium and will be called organothermal reactions in the following text. It should be stressed that the liquid used in reactions under pressure at temperatures over the normal boiling point of the liquid may act as a solvent or may take part in chemical reactions with the solids present in the heterogeneous mixture.

Compounds with open framework structures such as zeolites and aluminophosphates are traditionally obtained in hydrothermal synthesis from aqueous media and are in many cases only obtained as powders., ${ }^{1,2}$ However, it has been reported that the use of minor quantities of hydrofluoric acid as mineralizer in the hydrothermal synthesis has a drastic effect on the crystal sizes obtained in the hydrothermal synthesis, so that microporous compounds which previously could only be made as powders could now be obtained as crystals large enough for traditional single crystal X-ray analysis. ${ }^{3}$ It was also reported that crystals up to $1 \mathrm{~mm}$ in size of compounds with open framework structures can be

\footnotetext{
* To whom correspondence should be addressed.
}

obtained in organothermal synthesis in the temperature range $100-200^{\circ} \mathrm{C}$ using the organic solvents pyridine, triethylamine, polyethylene glycol ${ }^{4}$ and ethylene glycol. ${ }^{5}$ The use of organic solvents most likely results in a slower nucleation and crystal growth rate than in water, which then results in larger crystals in the organothermal synthesis.

A substantial amount of structural information can be gained from powder diffraction data, but more detailed structural information is obtained in single crystal diffraction analysis. It is thus tempting to improve the preparation techniques for the synthesis of microporous materials, and one obvious possibility is to use hydrofluoric acid as mineralizer in organothermal synthesis. The results of an investigation using hydrothermal and organothermal synthesis in the preparation of aluminophosphates and of $\mathrm{Zn}^{2+}$ - and $\mathrm{Co}^{2+}$-substituted aluminophosphates are reported below. Similar studies with $\mathrm{Mn}^{2+}$-substituted aluminophosphates are in progress and the results will be published elsewhere.

\section{Experimental}

Sample preparation. The amorphous gels used in the hydrothermal and organothermal synthesis were made from the following chemicals: $85 \% \mathrm{H}_{3} \mathrm{PO}_{4}, \mathrm{HF}$, $\mathrm{Zn}\left(\mathrm{CH}_{3} \mathrm{COO}\right)_{2} \cdot 2 \mathrm{H}_{2} \mathrm{O}$ and ethylene glycol (ETG) from Merck, and $98 \%$ aluminium isopropoxide, triethylamine 
(TEA), ethylenediamine (EDA), di- $n$-propylamine (DPA), tripropylamine (TPA), 1,4-diazabicyclo[2.2.2] octane (DABCO) and $\mathrm{Co}\left(\mathrm{CH}_{3} \mathrm{COO}\right)_{2} \cdot 4 \mathrm{H}_{2} \mathrm{O}$ from Aldrich. A $50-100 \mathrm{ml}$ charge was made of each gel. In the preparation of the ethylene glycol based gels, the procedure was as follows: The transition metal acetate and the aluminium isopropoxided were stirred with the ethylene glycol for approximately $1 \mathrm{~h}$. The $85 \% \mathrm{H}_{3} \mathrm{PO}_{4}$ was added and the mixture was stirred for at least $15 \mathrm{~min}$, and the organic amine was added with continuous stirring for at least $30 \mathrm{~min}$ to obtain homogeneity. The HF was finally added from a polyethylene pipette and the $\mathrm{pH}$ of the gels was measured using a $\mathrm{pH}$ meter. The charges were stored at room temperature in flasks of polyethylene. The gels with water as solvent were made in a similar way. Tables 1 and 2 list the composition of the gels and the experimental conditions for the synthesis. The ethylene glycol based gels are strictly speaking not purely non-aqueous, as they contain water from the $85 \% \mathrm{H}_{3} \mathrm{PO}_{4}$ and from the transition metal acetates. The products of the synthesis were washed with water and dried at room temperature. The structure types AFI, LEV and GIS mentioned in the two tables are described in Ref. 6 .

$x$-ray powder diffraction. X-Ray powder patterns of the products were recorded at $25^{\circ} \mathrm{C}$ on a Stoe-Stadi powder diffractometer with a position-sensitive detector. The diffractometer was calibrated with a silicon standard $(a=5.43050 \AA)$ and $\mathrm{Cu} K \alpha_{1}$ radiation was used $(\lambda=$ $1.5406 \AA$ ). Phases identified from the powder patterns and from X-ray single diffraction analysis are listed in Tables 1 and 2 .

$X$-Ray single crystal diffraction. Single crystals were selected using a polarizing microscope and were mounted on glass fibres with a two component glue. A Siemens SMART diffractometer with an area detector was applied using Mo $K \alpha$ radiation $(\lambda=0.7107 \AA)$. Crystallographic data are in Table 3 . The structures were solved using the program SIR, ${ }^{7}$ and were refined using the least-squares program LINUS $^{8}$ with scattering contributions from neutral atoms. ${ }^{9}$

\section{Results}

$\mathrm{Zn}^{2+}$-Substituted aluminophosphates, Table 1. The hydrothermal reactions of the $\mathrm{Zn}^{2+}$-substituted aluminophosphate gels gave with the templates TPA and DPA and hydrofluoric acid as mineralizer single crystals of ZnAPO-5, AFI, large enough for single crystal $\mathrm{X}$-ray analysis. The typical crystal size was $0.30 \times 0.08 \times 0.08 \mathrm{~mm}^{3}$. With the template DABCO crystalline ZnAPO-35. LEV. was obtained. The reaction products had crystals smaller than those obtained for ZnAPO-5 and an increase of the mineralizer concentration (experiment no. 260698 ) did not improve the crystal size significantly. When aluminium isopropylate was omitted in the reaction mixture (experiment no. 220 798), single crystals of $\mathrm{Zn}_{2} \mathrm{PO}_{4} \mathrm{~F}$ were obtained with typical sizes of $0.20 \times 0.05 \times 0.01 \mathrm{~mm}^{3}$. The $\mathrm{X}$-ray powder pat-

Table 1. Experimental conditions for hydrothermal $\left(\mathrm{H}_{2} \mathrm{O}\right)$ and organothermal $(\mathrm{ETG})$ syntheses of $\mathrm{Zn}^{2+}$-substituted aluminophosphates. $^{a}$

\begin{tabular}{|c|c|c|c|c|c|c|c|c|c|c|}
\hline $\begin{array}{l}\text { Sample } \\
\text { no. }\end{array}$ & Template & $\mathrm{MeO}$ & $\mathrm{Al}_{2} \mathrm{O}_{3}$ & $\mathrm{P}_{2} \mathrm{O}_{5}$ & $\mathrm{HF}$ & Solvent & $\begin{array}{l}\mathrm{pH} \\
\text { of gel }\end{array}$ & ${ }^{\mathrm{T} /}$ & $\begin{array}{l}\text { Time/ } \\
\mathbf{h}\end{array}$ & Product \\
\hline & TPA & $\mathrm{ZnO}$ & & & & $\mathrm{H}_{2} \mathrm{O}$ & & & & \\
\hline 031097 & $\begin{array}{l}1.60 \\
\text { DPA }\end{array}$ & 0.16 & 0.92 & 1.00 & 0.8 & 300 & 5.0 & 170 & 18 & ZnAPO-5, AFI \\
\hline 101097 & $\begin{array}{l}1.60 \\
\mathrm{DABCO}\end{array}$ & 0.40 & 0.80 & 1.00 & 0.8 & 300 & 5.0 & 170 & 18 & ZnAPO-5, AFI \\
\hline 261097 & 1.60 & 0.40 & 0.80 & 1.00 & 0.8 & 300 & 5.2 & 175 & 44 & ZnAPO-35, LEV \\
\hline 041197 & 1.60 & 0.40 & 0.80 & 1.00 & 0.8 & 300 & 5.5 & 170 & 48 & ZnAPO-35, LEV \\
\hline 020598 & 1.60 & 0.40 & 0.80 & 1.00 & 0.8 & 300 & 5.5 & 170 & 33 & ZnAPO-35, LEV \\
\hline 260698 & 1.60 & 0.40 & 0.80 & 1.00 & 1.9 & 300 & 5.3 & 180 & 69 & ZnAPO-35, LEV \\
\hline 220798 & $\begin{array}{l}1.60 \\
\text { EDA }\end{array}$ & 1.00 & & 1.00 & 1.90 & 300 & 4.7 & 180 & 103 & $\mathrm{Zn}_{2} \mathrm{PO}_{4} \mathrm{~F}$ \\
\hline 251097 & 1.60 & 0.40 & 0.80 & 1.00 & 1.33 & 300 & 5.2 & 175 & 25 & Not identified \\
\hline 041197 & 1.60 & 0.40 & 0.80 & 1.00 & 1.33 & 300 & 5.2 & 170 & 48 & Not identified \\
\hline $081197(\mathrm{~A})$ & 1.40 & 0.40 & 0.80 & 1.00 & 1.10 & 300 & 6.0 & 170 & 48 & Not identified \\
\hline $081197(B)$ & 1.40 & 0.40 & 0.80 & 1.00 & 1.10 & 300 & 6.0 & 170 & 48 & Not identified \\
\hline 030898 & 1.00 & 0.40 & 0.80 & 1.00 & 1.00 & 50 & 6.6 & 180 & 82 & Not identified \\
\hline 050598 & 2.00 & 0.42 & 0.13 & 1.00 & 1.65 & 138 & 5.8 & 180 & 96 & $(\mathrm{Zn}, \mathrm{Al}) \mathrm{PO}_{4} \cdot 0.5 \mathrm{C}_{2} \mathrm{H}_{10} \mathrm{~N}_{2}, \mathrm{ACP}-2$ \\
\hline 300698 & 2.00 & 0.70 & & 1.00 & 1.65 & $\begin{array}{l}138 \\
\text { ETG }\end{array}$ & 4.7 & 180 & 71 & $\mathrm{ZnPO}_{4} \cdot 0.5 \mathrm{C}_{2} \mathrm{H}_{10} \mathrm{~N}_{2}, \mathrm{ACP}-2$ \\
\hline 050398 & 1.75 & 0.25 & 0.50 & 1.00 & & 28 & 7.8 & 170 & 72 & Not identified \\
\hline 310798 & 1.75 & 0.25 & 0.50 & 1.00 & 1.3 & 56 & 5.9 & 180 & 72 & Not identified \\
\hline 030398 & 2.16 & 0.42 & 0.13 & 1.00 & & 33 & 6.7 & 180 & 74 & $(\mathrm{Zn}, \mathrm{Al}) \mathrm{PO}_{4} \cdot 0.5 \mathrm{C}_{2} \mathrm{H}_{10} \mathrm{~N}_{2}, \mathrm{ACP}-2$ \\
\hline 070498 & 2.16 & 0.42 & 0.13 & 1.00 & 2.50 & 33 & 4.8 & 180 & 96 & $(\mathrm{Zn}, \mathrm{Al}) \mathrm{PO}_{4} \cdot 0.5 \mathrm{C}_{2} \mathrm{H}_{10} \mathrm{~N}_{2}, \mathrm{ACP}-2$ \\
\hline 120598 & 2.00 & 0.70 & & 1.00 & 1.65 & 40 & 4.5 & 180 & 68 & $\mathrm{ZnPO}_{4} \cdot 0.5 \mathrm{C}_{2} \mathrm{H}_{10} \mathrm{~N}_{2}, \mathrm{ACP}-2$ \\
\hline
\end{tabular}

${ }^{a}$ Compositions of the reaction mixtures are listed as molar ratios. 
Table 2. Experimental conditions for organothermal (ETG) and hydrothermal $\left(\mathrm{H}_{2} \mathrm{O}\right)$ syntheses of $\mathrm{Co}^{2+}$-substituted aluminophosphates. $^{a}$

\begin{tabular}{|c|c|c|c|c|c|c|c|c|c|c|}
\hline $\begin{array}{l}\text { Sample } \\
\text { no. }\end{array}$ & Template & $\mathrm{MeO}$ & $\mathrm{Al}_{2} \mathrm{O}_{3}$ & $\mathrm{P}_{2} \mathrm{O}_{5}$ & HF & Solvent & $\begin{array}{l}\mathrm{pH} \\
\text { of gel }\end{array}$ & $\begin{array}{l}T / 1 \\
{ }^{\circ} \mathrm{C}\end{array}$ & $\begin{array}{l}\text { Time/ } \\
\mathrm{h}\end{array}$ & Product \\
\hline & TPA & $\mathrm{CoO}$ & & & & $\mathrm{H}_{2} \mathrm{O}$ & & & & \\
\hline 250997 & $\begin{array}{l}1.60 \\
\text { TEA }\end{array}$ & 0.16 & 0.92 & 1.00 & 0.8 & 300 & & 175 & 17 & CoAPO-5, AFI \\
\hline 160997 & 1.40 & 0.16 & 0.92 & 1.00 & & 40 & 6.5 & 195 & 70 & CoAPO-5, AFI \\
\hline 030798 & 1.40 & 0.16 & 0.92 & 1.00 & 1.55 & $\begin{array}{l}110 \\
\text { ETG }\end{array}$ & 5.4 & 180 & 119 & Berlinite \\
\hline 280298 & 1.00 & 0.11 & 0.65 & 1.00 & & 14 & 4.6 & 170 & 68 & CoAPO-5, AFI \\
\hline 070498 & 1.00 & 0.11 & 0.65 & 1.00 & 1.78 & 14 & 3.8 & 180 & 96 & CoAPO-5, AFI \\
\hline 190298 & 1.41 & 0.16 & 0.92 & 1.00 & & 11 & 6.5 & 175 & 65 & CoAPO-5, AFI \\
\hline 110498 & $\begin{array}{l}1.41 \\
\text { DABCO }\end{array}$ & 0.16 & 0.92 & 1.00 & 0.84 & $\begin{array}{r}11 \\
\mathrm{H}_{2} \mathrm{O}\end{array}$ & 6.3 & 180 & 96 & COAPO-5, AFI \\
\hline 270798 & $\begin{array}{l}1.90 \\
\text { EDA }\end{array}$ & 1.00 & & 1.00 & 1.90 & $\begin{array}{l}300 \\
\text { ETG }\end{array}$ & 5.2 & 180 & 82 & $\mathrm{Co}_{2} \mathrm{PO}_{4} \mathrm{~F}$ \\
\hline 060398 & 1.75 & 0.25 & 0.50 & 1.00 & & 28 & 7.4 & 170 & 72 & $\mathrm{CoAl}\left(\mathrm{PO}_{4}\right)_{2} \cdot \mathrm{C}_{2} \mathrm{H}_{9} \mathrm{~N}_{2}$ \\
\hline $110498-A$ & 1.25 & 0.25 & 0.75 & 1.00 & & 30 & 6.9 & 180 & 96 & $\mathrm{CoAl}\left(\mathrm{PO}_{4}\right)_{2} \cdot \mathrm{C}_{2} \mathrm{H}_{9} \mathrm{~N}_{2}$ \\
\hline 210298 & 2.16 & 0.84 & 0.13 & 1.00 & & 33 & 5.7 & 180 & 140 & $\begin{array}{l}(\mathrm{Co}, \mathrm{Al}) \mathrm{PO}_{4} \cdot 0.5 \mathrm{C}_{2} \mathrm{H}_{10} \mathrm{~N}_{2}, \mathrm{ACP}-3 \\
\text { and CoAl }\left(\mathrm{PO}_{4}\right)_{2} \cdot \mathrm{C}_{2} \mathrm{H}_{9} \mathrm{~N}_{2}\end{array}$ \\
\hline 280298 & 2.16 & 0.42 & 0.13 & 1.00 & & 33 & 6.2 & 180 & 69 & $\begin{array}{l}\text { (Co,Al) } \mathrm{PO}_{4} \cdot 0.5 \mathrm{C}_{2} \mathrm{H}_{10} \mathrm{~N}_{2}, \mathrm{ACP}-3 \\
\text { and CoAl }\left(\mathrm{PO}_{4}\right)_{2} \cdot \mathrm{C}_{2} \mathrm{H}_{9} \mathrm{~N}_{2}\end{array}$ \\
\hline 170498 & 2.00 & 0.42 & 0.13 & 1.00 & & 40 & 6.3 & 180 & 96 & $(\mathrm{Co}, \mathrm{Al}) \mathrm{PO}_{4} \cdot 0.5 \mathrm{C}_{2} \mathrm{H}_{10} \mathrm{~N}_{2}, \mathrm{ACP}-3$ \\
\hline 150698 & 2.00 & 0.70 & & 1.00 & & $\begin{array}{r}40 \\
\mathrm{H}_{2} \mathrm{O}\end{array}$ & 5.4 & 180 & 72 & $\mathrm{CoPO}_{4} \cdot 0.5 \mathrm{C}_{2} \mathrm{H}_{10} \mathrm{~N}_{2}, \mathrm{ACP}-3$ \\
\hline \multirow[t]{2}{*}{020598} & 2.00 & 0.42 & 0.13 & 1.00 & & 138 & 6.8 & 180 & 70 & $\begin{array}{l}\mathrm{CoAl}\left(\mathrm{PO}_{4}\right)_{2} \cdot \mathrm{C}_{2} \mathrm{H}_{9} \mathrm{~N}_{2} \\
\text { and } \mathrm{CoAPO}-43 \text {, GIS }\end{array}$ \\
\hline & & & & & & ETG & & & & \\
\hline $070498-4$ & 1.75 & 0.25 & 0.50 & 1.00 & 2.24 & 28 & 6.2 & 180 & 96 & Not identified \\
\hline 110498 & 1.00 & 0.20 & 0.60 & 1.00 & 0.32 & 24 & 4.5 & 180 & 96 & X-Ray amorphous \\
\hline $070498-2$ & 2.16 & 0.84 & 0.13 & 1.00 & 2.34 & 33 & 5.1 & 180 & 96 & $\begin{array}{l}\text { CoAPO-43, GIS and } \\
(\mathrm{Co}, \mathrm{Al}) \mathrm{PO}_{4} \cdot 0.5 \mathrm{C}_{2} \mathrm{H}_{10} \mathrm{~N}_{2}, \mathrm{ACP}-3\end{array}$ \\
\hline 110498 & 1.96 & 0.55 & 0.32 & 1.00 & 2.29 & 31 & 5.3 & 180 & 96 & Not identified \\
\hline $070498-S$ & 2.16 & 0.42 & 0.13 & 1.00 & 1.56 & 33 & 5.6 & 180 & 96 & COAPO- 43 , GIS \\
\hline 220498 & 2.00 & 0.42 & 0.13 & 1.00 & 1.65 & 40 & 5.4 & 180 & 103 & COAPO- 43 , GIS \\
\hline 100698 & 2.00 & 0.70 & & 1.00 & 1.65 & $\begin{array}{r}40 \\
\mathrm{H}_{2} \mathrm{O}\end{array}$ & 5.1 & 180 & 103 & $\mathrm{CoPO}_{4} \cdot 0.5 \mathrm{C}_{2} \mathrm{H}_{10} \mathrm{~N}_{2}$, ACP-3 \\
\hline 280498 & 2.00 & 0.42 & 0.13 & 1.00 & 1.65 & 138 & 6.0 & 180 & 84 & CoAPO- 43 , GIS \\
\hline
\end{tabular}

${ }^{a}$ Compositions of the reaction mixtures are listed as molar ratios.

Table 3. Crystallographic data for CoAIPO ${ }_{4} \cdot \mathrm{C}_{2} \mathrm{H}_{9} \mathrm{~N}_{2}, \mathrm{CoPO}_{4} \cdot 0.5 \mathrm{C}_{2} \mathrm{H}_{10} \mathrm{~N}_{2}$ and CoPO $\mathrm{CO}_{4} \cdot 0.5 \mathrm{C}_{2} \mathrm{H}_{10} \mathrm{~N}_{2} \cdot 0.5 \mathrm{H}_{2} \mathrm{O}$.

\begin{tabular}{llll}
\hline & $\mathrm{CoAIPO}_{4} \cdot \mathrm{C}_{2} \mathrm{H}_{9} \mathrm{~N}_{2}$ & $\begin{array}{l}\mathrm{CoPO}_{4} \cdot 0.5 \mathrm{C}_{2} \mathrm{H}_{10} \mathrm{~N}_{2} \\
(\mathrm{ACP}-3)\end{array}$ & $\begin{array}{l}(\mathrm{Co}, \mathrm{Al}) \mathrm{PO}_{4} \cdot 0.5 \mathrm{C}_{2} \mathrm{H}_{10} \mathrm{~N}_{2} \cdot 0.5 \mathrm{H}_{2} \mathrm{O} \\
\left(\mathrm{CoAPO}-43, \mathrm{GIS}^{2}\right.\end{array}$ \\
\hline$a / \AA$ & $8.6026(6)$ & $10.4123(6)$ & $10.1724(6)$ \\
$b / \AA$ & $15.5520(11)$ & & \\
$c / \AA$ & $7.7436(6)$ & $8.9477(8)$ & $9.6060(6)$ \\
$\beta /{ }^{\circ}$ & $110.634(1)$ & & \\
$V / \AA^{3}$ & $969.5(1)$ & $970.1(1)$ & $994.0(1)$ \\
$Z$ & 4 & 8 & 8 \\
Space group & $P 21 / c$ & $P 44_{2} / n$ & $1 \overline{4} 2 \mathrm{~m}$ \\
Unique data & 1324 & 563 & $0.05 \times 0.05 \times 0.05$ \\
Crystal size/mm & $0.10 \times 0.10 \times 0.01$ & $0.08 \times 0.08 \times 0.05$ & 54 \\
Parameters & 146 & 73 & 5.7 \\
$R(F)(\%)$ & 4.2 & 3.5 & 7.9 \\
$R_{w}(F)(\%)$ & 5.6 & 5.2 & \\
\hline
\end{tabular}

${ }^{a}$ The number of reflections with $I>3 \sigma(l)$ are listed as unique data. Data collection at $25^{\circ} \mathrm{C}$. 
tern of $\mathrm{ZnAPO}-35, \mathrm{LEV}$, the reaction product of no. 260698 is listed in Table 4. It was indexed with the program DICVOL91 ${ }^{10}$ on a hexagonal cell with $a=$ 13.234(9) and $c=22.31$ (2) $\AA$, which is similar to the unit cell for Levyne. ${ }^{11}$ The powder pattern is very similar to the unindexed pattern of aluminium silicon phosphate quinuclidine, ICDD card no. 47-0062. This pattern was also indexed with the program DICVOL9 ${ }^{10}$ on a hexagonal cell with $a=13.255(7)$ and $c=22.36(2) \AA$, and observed and calculated values are listed in Table 5 .

The hydrothermal and organothermal synthesis with EDA as the template and a low $\mathrm{ZnO} / \mathrm{Al}_{2} \mathrm{O}_{3}$ ratio gave products which had powder patterns with many lines

Table 4. Powder pattern of ZnAPO-35, LEV, sample no. 260698 , Table 1. ${ }^{a}$

\begin{tabular}{rrrrrrrr}
\hline $2 \theta_{\text {obs }}$ & $2 \theta_{\text {calc }}$ & \multicolumn{1}{c}{$d_{\text {obs }}$} & \multicolumn{1}{c}{$d_{\text {calc }}$} & $l_{\text {obs }}$ & $h$ & $k$ & $l$ \\
\hline 8.60 & 8.67 & 10.27 & 10.19 & 20 & 1 & 0 & 1 \\
11.05 & 11.06 & 8.00 & 7.99 & 53 & 1 & 0 & 2 \\
11.87 & 11.89 & 7.44 & 7.44 & 15 & 0 & 0 & 3 \\
13.37 & 13.37 & 6.617 & 6.616 & 41 & 1 & 1 & 0 \\
15.96 & 15.96 & 5.548 & 5.555 & 18 & 2 & 0 & 1 \\
17.38 & 17.38 & 5.098 & 5.097 & 79 & 2 & 0 & 2 \\
17.65 & 17.67 & 5.020 & 5.015 & 21 & 1 & 0 & 4 \\
21.32 & 21.35 & 4.164 & 4.158 & 37 & 1 & 0 & 5 \\
21.98 & 21.99 & 4.040 & 4.038 & 100 & 2 & 1 & 2 \\
23.24 & 23.27 & 3.824 & 3.820 & 37 & 3 & 0 & 0 \\
23.80 & 23.75 & 3.735 & 3.743 & 10 & 2 & 1 & 3 \\
25.20 & 25.16 & 3.531 & 3.537 & 27 & 1 & 0 & 6 \\
26.95 & 26.93 & 3.305 & 3.308 & 33 & 2 & 2 & 0 \\
28.70 & 28.70 & 3.108 & 3.108 & 26 & 2 & 1 & 5 \\
32.23 & 32.23 & 2.775 & 2.775 & 54 & 4 & 0 & 2 \\
\hline
\end{tabular}

Indexed with the hexagonal unit cell $a=13.294(9), c=$ 22.31(2) A. Figure of merit $M(15)=11.0$.

Table 5. Powder pattern of aluminium silicon phosphate quinuclidine, ICDD card no. 47-0622. ${ }^{a}$

\begin{tabular}{rrcrrrrr}
\hline $2 \theta_{\text {obs }}$ & $2 \theta_{\text {calc }}$ & \multicolumn{1}{l}{$d_{\text {obs }}$} & \multicolumn{1}{l}{$d_{\text {calc }}$} & $l_{\text {obs }}$ & $h$ & $k$ & $l$ \\
\hline 8.66 & 8.65 & $10.20^{b}$ & 10.212 & 18 & 1 & 0 & 1 \\
11.04 & 11.04 & 8.01 & 8.009 & 47 & 1 & 0 & 2 \\
11.89 & 11.86 & 7.44 & 7.454 & 2 & 0 & 0 & 3 \\
13.38 & 13.35 & 6.61 & 6.627 & 13 & 1 & 1 & 0 \\
15.99 & 15.93 & 5.54 & 5.559 & 12 & 2 & 0 & 1 \\
17.37 & 17.35 & 5.10 & 5.106 & 83 u & 2 & 0 & 2 \\
17.69 & 17.63 & 5.01 & 5.026 & $u$ & 1 & 0 & 4 \\
17.87 & 17.89 & 4.96 & 4.953 & 14 & 1 & 1 & 3 \\
21.24 & 21.30 & 4.18 & 4.167 & 55 & 1 & 0 & 5 \\
21.98 & 21.96 & 4.04 & 4.044 & 100 & 2 & 1 & 2 \\
23.27 & 23.23 & 3.82 & 3.826 & 18 & 3 & 0 & 0 \\
23.71 & 23.71 & 3.75 & 3.749 & 6 & 2 & 1 & 3 \\
25.21 & 25.22 & 3.53 & 3.527 & 5 & 2 & 0 & 5 \\
25.98 & 25.97 & 3.427 & 3.427 & 1 & 2 & 1 & 4 \\
26.88 & 26.88 & 3.314 & 3.313 & 18 & 2 & 2 & 0 \\
26.53 & 26.52 & 3.126 & 3.127 & 26 & 2 & 0 & 6 \\
28.68 & 28.64 & 3.110 & 3.114 & 13 & 2 & 1 & 5 \\
29.07 & 29.14 & 3.069 & 3.062 & 6 & 3 & 1 & 2 \\
32.13 & 32.18 & 2.784 & 2.779 & 40 & 4 & 0 & 2 \\
\hline
\end{tabular}

${ }^{a}$ Hexagonal unit cell $a=13.255(7), c=22.36(2) \AA$. Figure of merit $M(19)=7.8$. ${ }^{b}$ The $d$-value listed on the card was $10.10 \AA$. $2 \theta_{\text {obs }}$ corresponds to the $d$-values on the card for Cu $K \alpha_{1}$ radiation $(\lambda=1.5406 \AA$ ). difficult to index. With a high $\mathrm{ZnO} / \mathrm{Al}_{2} \mathrm{O}_{3}$ ratio crystalline products were obtained which gave few lines. The X-ray powder pattern of the reaction product from experiment no. 070498 is listed in Table 6 . The pattern was indexed with the program DICVOL9 $1^{10}$ on an orthorhombic unit cell with $a=14.80(1), b=14.75(1), c=8.99$ (1) $\AA$, which is similar to the unit cell of ACP- $2,{ }^{12}$ and the product could possibly have a structure similar to that of ACP2. When aluminium isopropylate was omitted from the reaction mixture, the product in the hydrothermal experiment (no. 300698) and the organothermal synthesis (experiment no. 120598) was an ACP-2 type phase with the composition $\mathrm{ZnPO}_{4} \cdot 0.5 \mathrm{C}_{2} \mathrm{H}_{10} \mathrm{~N}_{2}$.

$\mathrm{Co}^{2+}$-Substituted aluminophosphates, Table 2. The $\mathrm{Co}^{2+}$ substituted aluminophosphate gels gave with the templates TPA and TEA crystalline samples of CoAPO-5, AFI. The use of hydrofluoric acid as mineralizer gave single crystals of the product with sizes up to $0.30 \times 0.05 \times 0.05 \mathrm{~mm}^{3}$. In the organothermal synthesis with the template solvent combination TEA-ETG crystalline CoAPO-5 was obtained, but the use of hydrofluoric acid did not improve the crystal size of the product. With EDA as the structure-directing amine the organothermal and hydrothermal synthesis gave

Table 6. Powder pattern of $(\mathrm{Zn}, \mathrm{Al}) \mathrm{PO}_{4} \cdot 0.5 \mathrm{C}_{2} \mathrm{H}_{10} \mathrm{~N}_{2}, \mathrm{ACP}-2$, sample no. 070498 , Table $1 .^{a}$

\begin{tabular}{llllllll}
\hline $2 \theta_{\text {obs }}$ & $2 \theta_{\text {calc }}$ & $d_{\text {obs }}$ & $d_{\text {calc }}$ & $I_{\text {obs }}$ & $h$ & $k$ & $l$ \\
\hline 11.90 & 11.95 & 7.433 & 7.401 & 87 & 2 & 0 & 0 \\
15.46 & 15.50 & 5.727 & 5.714 & 37 & 2 & 0 & 1 \\
16.91 & 16.96 & 5.238 & 5.225 & 18 & 2 & 2 & 0 \\
19.69 & 19.64 & 4.505 & 4.517 & 27 & 2 & 2 & 1 \\
& 19.73 & & 4.495 & & 0 & 0 & 2 \\
21.46 & 21.44 & 4.137 & 4.142 & 24 & 1 & 3 & 1 \\
23.11 & 23.13 & 3.846 & 3.842 & 44 & 2 & 0 & 2 \\
24.80 & 24.78 & 3.587 & 3.590 & 10 & 4 & 1 & 0 \\
26.04 & 26.02 & 3.419 & 3.422 & 100 & 4 & 0 & 1 \\
26.95 & 26.99 & 3.306 & 3.301 & 97 & 2 & 4 & 0 \\
& 26.93 & & 3.308 & & 4 & 2 & 0 \\
28.74 & 28.74 & 3.104 & 3.104 & 11 & 4 & 2 & 1 \\
30.15 & 30.20 & 2.962 & 2.957 & 7 & 4 & 3 & 0 \\
32.21 & 32.20 & 2.777 & 2.778 & 26 & 2 & 0 & 3 \\
& 32.22 & & 2.776 & & 0 & 2 & 3 \\
32.57 & 32.56 & 2.747 & 2.748 & 18 & 5 & 2 & 0 \\
33.63 & 33.61 & 2.663 & 2.664 & 19 & 4 & 2 & 2 \\
34.30 & 34.30 & 2.612 & 2.612 & 28 & 4 & 4 & 0 \\
35.58 & 35.57 & 2.521 & 2.522 & 9 & 1 & 3 & 3 \\
& 35.55 & & 2.524 & & 3 & 1 & 3 \\
36.51 & 36.52 & 2.459 & 2.459 & 6 & 0 & 6 & 0 \\
37.12 & 37.15 & 2.420 & 2.419 & 7 & 2 & 3 & 3 \\
38.68 & 37.13 & & 2.420 & & 3 & 2 & 3 \\
& 38.63 & 2.326 & 2.329 & 22 & 4 & 0 & 3 \\
40.64 & 40.58 & & 2.326 & & 0 & 4 & 3 \\
& 40.63 & 2.218 & 2.220 & 15 & 4 & 2 & 3 \\
40.88 & 40.87 & 2.206 & 2.219 & & 2 & 4 & 3 \\
& 40.82 & & 2.206 & 15 & 3 & 5 & 2 \\
43.67 & 43.67 & 2.071 & 2.071 & 8 & 2 & 6 & 2 \\
\hline
\end{tabular}

${ }^{a}$ Indexed with the orthorhombic unit cell $a=14.80(1), b=$ $14.75(1), c=8.99(1) \AA$. Figure of merit $M(22)=7.1$. 
two main products, $\operatorname{CoAl}\left(\mathrm{PO}_{4}\right)_{2} \cdot \mathrm{C}_{2} \mathrm{H}_{9} \mathrm{~N}_{2}$ and $(\mathrm{Co}, \mathrm{Al}) \mathrm{PO}_{4} \cdot 0.5 \mathrm{C}_{2} \mathrm{H}_{10} \mathrm{~N}_{2}, \mathrm{ACP}-3 .{ }^{13}$ The crystal sizes were $0.15 \times 0.15 \times 0.03$ and $0.10 \times 0.10 \times 0.05$, respectively. The X-ray diffraction powder pattern of this ACP3 type compound, the reaction product of no. 170498, is listed in Table 7. Indexing with DICVOL $91^{10}$ gave a tetragonal unit cell with $a=10.443(5)$ and $c=9.008(6) \AA$. This unit cell has only half the volume of that of the cell for the $\mathrm{Zn}^{2+}$-substituted aluminophosphate described in Table 6. A powder pattern similar to that of Table 7 was measured for the reaction product of no. 150698. This synthesis did not contain aluminium in the reaction mixture, so the composition of the product is $\mathrm{CoPO}_{4} \cdot 0.5 \mathrm{C}_{2} \mathrm{H}_{10} \mathrm{~N}_{2}$, ACP-3.

The X-ray diffraction powder pattern of $\mathrm{CoAl}\left(\mathrm{PO}_{4}\right)_{2} \cdot \mathrm{C}_{2} \mathrm{H}_{9} \mathrm{~N}_{2}$ the product of no. 020598 was indexed with DICVOL91 ${ }^{10}$ and gave a monoclinic unit cell with $a=8.62(2), b=15.55(5), c=7.79(3) \AA$ and $\beta=$ $111.0(2)^{\circ}$ (Table 8). This is in good agreement with the unit cell reported in the single crystal structure investigation of $\mathrm{CoAl}\left(\mathrm{PO}_{4}\right)_{2} \cdot \mathrm{C}_{2} \mathrm{H}_{9} \mathrm{~N}_{2} \cdot{ }^{14}$

The X-ray diffraction powder pattern of the product of the hydrothermal synthesis of experiment no. 280498 is listed in Table 9. Indexing with DICVOL9 ${ }^{10}$ gave a tetragonal unit cell with $a=10.21(2)$ and $c=9.64(1) \AA$, which is close to the size of the unit cell for the magnesium substituted aluminophosphate MgAPO- $43^{15}$ with a structure of the gismondine type. The organothermal and hydrothermal synthesis thus gave with EDA as structuredirecting amine and hydrofluoric acid as mineralizer a gismondine-like phase and ACP-2 as the main products.

Crystal structures from single crystal diffractometry. The data analysis of the single crystal diffraction data for $\mathrm{CoAl}\left(\mathrm{PO}_{4}\right)_{2} \cdot \mathrm{C}_{2} \mathrm{H}_{9} \mathrm{~N}_{2}$ and $(\mathrm{Co}, \mathrm{Al}) \mathrm{PO}_{4} \cdot 0.5 \mathrm{C}_{2} \mathrm{H}_{10} \mathrm{~N}_{2}$ confirmed the previously reported structures. ${ }^{13,14}$ The number of reflections was not sufficiently large to improve

Table 7. Powder pattern of $(\mathrm{Co}, \mathrm{Al}) \mathrm{PO}_{4} \cdot 0.5 \mathrm{C}_{2} \mathrm{H}_{10} \mathrm{~N}_{2}$, ACP-3, sample no. 170498 , Table $2 .^{a}$

\begin{tabular}{|c|c|c|c|c|c|c|c|c|c|c|}
\hline $2 \theta_{\text {obs }}$ & $2 \theta_{\text {calc }}$ & $d_{\mathrm{obs}}$ & $d_{\text {calc }}$ & $I_{\mathrm{obs}}$ & $h$ & $k$ & 1 & & & \\
\hline 11.90 & 11.98 & 7.431 & 7.384 & 36 & 1 & 1 & 0 & & & \\
\hline 15.47 & 15.50 & 5.723 & 5.711 & 26 & 1 & 1 & 1 & & & \\
\hline 16.98 & 16.97 & 5.218 & 5.232 & 5 & 2 & 0 & 0 & & & \\
\hline 19.68 & 19.69 & 4.507 & 4.504 & 29 & 0 & 0 & 2 & & & \\
\hline 21.43 & 21.46 & 4.143 & 4.146 & 13 & 1 & 0 & 2 & & & \\
\hline 23.09 & 23.12 & 3.849 & 3.845 & 43 & 1 & 1 & 2 & & & \\
\hline 26.06 & 26.06 & 3.417 & 3.416 & 100 & 2 & 2 & 1 & & & \\
\hline 26.97 & 26.98 & 3.303 & 3.302 & 45 & 1 & 3 & 0 & 3 & 1 & 0 \\
\hline 28.72 & 28.77 & 3.106 & 3.101 & 17 & 1 & 3 & 1 & 3 & 1 & 1 \\
\hline 32.16 & 32.15 & 2.781 & 2.782 & 21 & 1 & 1 & 3 & & & \\
\hline 32.46 & 32.44 & 2.756 & 2.757 & 10 & 2 & 3 & 1 & 3 & 2 & 1 \\
\hline 33.64 & 33.62 & 2.662 & 2.663 & 17 & 1 & 3 & 2 & 3 & 1 & 2 \\
\hline 34.37 & 34.32 & 2.607 & 2.611 & 14 & 4 & 0 & 0 & & & \\
\hline 35.51 & 35.51 & 2.526 & 2.526 & 6 & 2 & 1 & 3 & 1 & 2 & 3 \\
\hline 38.63 & 38.62 & 2.329 & 2.330 & 21 & 2 & 2 & 3 & & & \\
\hline 40.58 & 40.57 & 2.221 & 2.222 & 13 & 3 & 1 & 3 & 1 & 3 & 3 \\
\hline
\end{tabular}

${ }^{a}$ Indexed with the tetragonal unit cell $a=10.443(5), c=$ $9.008(6) \AA ̊$. Figure of merit $M(16)=18.1$.
Table 8. Powder pattern of $\mathrm{CoAl}\left(\mathrm{PO}_{4}\right)_{2} \cdot \mathrm{C}_{2} \mathrm{H}_{9} \mathrm{~N}_{2}$, sample no. 020598 , Table $2 .^{a}$

\begin{tabular}{llllrllr}
\hline $2 \theta_{\text {obs }}$ & $2 \theta_{\text {calc }}$ & $d_{\text {obs }}$ & $d_{\text {calc }}$ & $I_{\text {obs }}$ & $h$ & $k$ & $I$ \\
\hline 10.90 & 10.89 & 8.110 & 8.116 & 6 & 1 & 0 & 0 \\
11.30 & 11.35 & 7.824 & 7.790 & 10 & 0 & 2 & 0 \\
12.27 & 12.29 & 7.208 & 7.198 & 100 & 1 & 1 & 0 \\
13.40 & 13.42 & 6.602 & 6.595 & 76 & 0 & 1 & 1 \\
17.40 & 17.43 & 5.093 & 5.084 & 9 & 1 & 2 & -1 \\
20.31 & 20.28 & 4.369 & 4.374 & 8 & 1 & 3 & 0 \\
20.96 & 21.00 & 4.235 & 4.228 & 9 & 0 & 3 & 1 \\
21.76 & 21.76 & 4.081 & 4.082 & 5 & 2 & 1 & -1 \\
22.22 & 22.19 & 3.998 & 4.004 & 27 & 1 & 2 & 1 \\
22.73 & 22.81 & 3.909 & 3.895 & 8 & 0 & 4 & 0 \\
22.97 & 23.00 & 3.869 & 3.864 & 4 & 1 & 0 & -2 \\
23.70 & 23.70 & 3.751 & 3.751 & 4 & 1 & 1 & -2 \\
24.47 & 24.44 & 3.635 & 3.640 & 14 & 0 & 0 & 2 \\
25.39 & 25.34 & 3.505 & 3.512 & 2 & 1 & 4 & 0 \\
27.04 & 27.02 & 3.295 & 3.298 & 6 & 0 & 2 & 2 \\
27.16 & 27.17 & 3.281 & 3.280 & 21 & 2 & 3 & -1 \\
28.77 & 28.77 & 3.101 & 3.100 & 39 & 1 & 3 & -2 \\
29.17 & 29.25 & 3.059 & 3.051 & 4 & 2 & 1 & 1 \\
30.76 & 30.75 & 2.904 & 2.905 & 7 & 1 & 1 & 2 \\
21.89 & 31.82 & 2.804 & 2.810 & 22 & 2 & 4 & 0 \\
33.06 & 33.06 & 2.707 & 2.707 & 11 & 3 & 2 & -1 \\
33.71 & 33.68 & 2.657 & 2.659 & 3 & 0 & 4 & 2 \\
35.28 & 35.28 & 2.542 & 2.542 & 4 & 2 & 4 & -2 \\
\hline
\end{tabular}

${ }^{a}$ Indexed with the monoclinic unit cell $a=8.66(1), b=$ 15.58(2), $c=7.77(1) \AA, \beta=110.5(1)^{\circ}$. Values for the structure of $\mathrm{CoAl}\left(\mathrm{PO}_{4}\right)_{2} \cdot \mathrm{C}_{2} \mathrm{H}_{9} \mathrm{~N}_{2}$ are: $a=8.603(1), b=15.552(1), c=$ $7.744(1) \AA, \beta=110.60(1)^{\circ}$. Figure of merit $M(23)=6.6$.

Table 9. Powder pattern of $(\mathrm{Co}, \mathrm{Al}) \mathrm{PO}_{4} \cdot 0.5 \mathrm{C}_{2} \mathrm{H}_{10} \mathrm{~N}_{2} \cdot 0.5 \mathrm{H}_{2} \mathrm{O}$, CoAPO-43, GIS, sample no. 280498 , Table 2. ${ }^{a}$

\begin{tabular}{ccccrlll}
\hline $2 \theta_{\text {obs }}$ & $2 \theta_{\text {calc }}$ & $d_{\text {obs }}$ & $d_{\text {calc }}$ & $l_{\text {obs }}$ & $h$ & $k$ & $l$ \\
\hline 12.22 & 12.30 & 7.240 & 7.191 & 46 & 1 & 1 & 0 \\
12.61 & 12.66 & 7.017 & 6.983 & 100 & 1 & 0 & 1 \\
17.34 & 17.42 & 5.109 & 5.085 & 12 & 2 & 0 & 0 \\
18.45 & 18.46 & 4.804 & 4.803 & 7 & 0 & 0 & 2 \\
21.49 & 21.60 & 4.132 & 4.111 & 8 & 2 & 1 & 1 \\
22.17 & 22.44 & 4.007 & 3.994 & 20 & 1 & 1 & 2 \\
25.41 & 25.48 & 3.502 & 3.492 & 47 & 2 & 0 & 2 \\
27.73 & 27.72 & 3.215 & 3.216 & 71 & 3 & 1 & 0 \\
& 27.88 & & 3.197 & & 3 & 0 & 1 \\
29.16 & 29.22 & 3.060 & 3.054 & 33 & 1 & 0 & 3 \\
30.95 & 31.04 & 2.887 & 2.878 & 14 & 2 & 2 & 2 \\
32.94 & 33.08 & 2.717 & 2.706 & 9 & 3 & 2 & 1 \\
33.37 & 33.50 & 2.683 & 2.672 & 19 & 3 & 1 & 2 \\
34.12 & 34.22 & 2.625 & 2.688 & 18 & 2 & 1 & 3 \\
37.37 & 37.48 & 2.404 & 2.397 & 11 & 3 & 3 & 0 \\
38.52 & 37.62 & & 2.389 & & 4 & 1 & 1 \\
38.64 & 2.335 & 2.328 & 7 & 3 & 0 & 3 \\
\hline
\end{tabular}

${ }^{a}$ Indexed with a tetragonal unit cell $a=10.21(2), c=9.64(1) \AA$. Values from the single crystal structure analysis of $\left(\mathrm{Co}_{0.85} \mathrm{Al}_{0.15}\right) \mathrm{PO}_{4} \cdot 0.5 \mathrm{C}_{2} \mathrm{H}_{10} \mathrm{~N}_{2} \cdot 0.5 \mathrm{H}_{2} \mathrm{O}$ were $\mathrm{a}=10.1724(6)$, $c=9.6060(6) \AA$, space group $I \overline{4} 2 \mathrm{~m}$ and were used to calculate $d_{\text {calc }}$ and $2 \theta_{\text {calc }}$. Figure of merit $M(10)=17.5$.

the structures, and the results were only used to calculate the powder patterns listed in Table 8 using the program LAZY PULVERIX. ${ }^{16}$ The size of the unit cell of $(\mathrm{Co}, \mathrm{Al}) \mathrm{PO}_{4} \cdot 0.5 \mathrm{C}_{2} \mathrm{H}_{10} \mathrm{~N}_{2}$ is thus half of that found for $(\mathrm{Zn}, \mathrm{Al}) \mathrm{PO}_{4} \cdot 0.5 \mathrm{C}_{2} \mathrm{H}_{10} \mathrm{~N}_{2}$ and that reported previously 
Table 10. Atomic coordinates and displacement parameters $\left(\times 10^{4}\right)$ for $\left(\mathrm{Co}_{0.84} \mathrm{Al}_{0.16}\right) \mathrm{PO}_{4} \cdot 0.5 \mathrm{C}_{2} \mathrm{H}_{10} \mathrm{~N}_{2} \cdot 0.5 \mathrm{H}_{2} \mathrm{O}, R=5.7 \%$.

\begin{tabular}{llllllllrr}
\hline Atom & $x / a$ & $y / b$ & $z / c$ & $U_{11}$ & $U_{22}$ & $U_{33}$ & $U_{12}$ & $U_{13}$ & $U_{23}$ \\
\hline Co,Al & $0.8668(1)$ & $0.1332(1)$ & $0.3684(2)$ & $71(2)$ & $71(2)$ & $130(3)$ & $8(2)$ & $-18(2)$ & $18(2)$ \\
P & $0.6646(3)$ & $0.3354(3)$ & $0.1918(4)$ & $84(3)$ & $84(3)$ & $124(3)$ & $23(3)$ & $-18(3)$ & $18(3)$ \\
O1 & $0.7001(9)$ & $0.2999(1)$ & $0.038(2)$ & $138(10)$ & $138(10)$ & $201(24)$ & $43(16)$ & $30(10)$ & $-30(10)$ \\
O2 & $1.020(1)$ & $0.1969(9)$ & $0.2816(9)$ & $118(11)$ & $126(10)$ & $183(10)$ & $-5(10)$ & $-14(11)$ & $88(10)$ \\
O3 & $0.742(2)$ & $0.259(1)$ & $0.286(3)$ & $231(16)$ & $231(16)$ & $401(50)$ & $34(21)$ & $-154(2)$ & $154(23)$ \\
O4 & 0 & 0 & $1 / 2$ & $126(20)$ & $126(20)$ & $150(29)$ & 0 & 0 & 0 \\
O5 & 0 & 0 & 0 & $167(25)$ & $167(25)$ & $206(40)$ & 0 & 0 & 0 \\
C & $0.504(2)$ & $0.050(2)$ & $0.435(2)$ & $48(14)$ & $92(19)$ & $66(15)$ & $8(24)$ & $0(17)$ & $-2(16)$ \\
N & $1 / 2$ & $0.185(2)$ & $1 / 2$ & $74(13)$ & $86(14)$ & $165(26)$ & 0 & $-6(15)$ & 0 \\
\hline
\end{tabular}
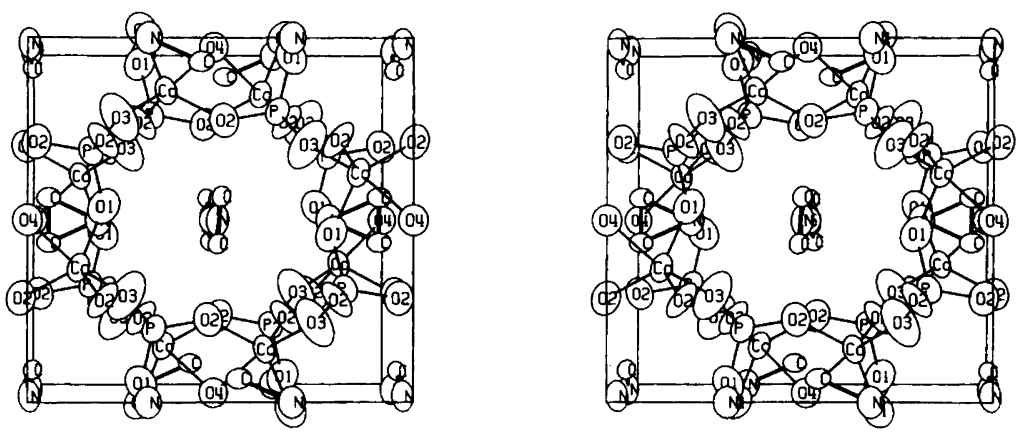

Fig. 1. Stereoscopic drawing of the structure of $\left(\mathrm{Co}_{0.84} \mathrm{Al}_{0.16}\right) \mathrm{PO}_{4} \cdot 0.5 \mathrm{C}_{2} \mathrm{H}_{10} \mathrm{~N}_{2} \cdot 0.5 \mathrm{H}_{2} \mathrm{O}$ along [001].

for DAF-2. ${ }^{17}$ A single crystal analysis of the product from experiment no. 280498 showed that the crystal had a gismondine-type structure and the composition $\left(\mathrm{Co}_{0.84} \mathrm{Al}_{0.16}\right) \mathrm{PO}_{4} \cdot 0.5 \mathrm{C}_{2} \mathrm{H}_{10} \mathrm{~N}_{2} \cdot 0.5 \mathrm{H}_{2} \mathrm{O}$ (Table 10). It is thus a hydrate obtained in an organothermal synthesis. It was pointed out previously that the syntheses are not non-aqueous, strictly speaking. A list of interatomic distances is given in Table 11.

The cobalt and aluminium content of the sample was deduced from a consideration of the metal-oxygen atoms in the structure, in a linear interpolation between the $\mathrm{Co}-\mathrm{O}$ and $\mathrm{Al}-\mathrm{O}$ distances 1.93 and $1.74 \AA$, respectively. ${ }^{12}$ Refinement of the site occupancy of the cobalt atom indicated that this site contained $10-15 \%$ aluminium. Figure 1 shows a stereoscopic drawing of the

Table 11. Interatomic distances (in $\AA$ ) of $\left(\mathrm{Co}_{0.84} \mathrm{Al}_{0.16}\right) \mathrm{PO}_{4}$. $0.5 \mathrm{C}_{2} \mathrm{H}_{10} \mathrm{~N}_{2} \cdot 0.5 \mathrm{H}_{2} \mathrm{O}^{a}$

\begin{tabular}{|c|c|c|}
\hline $\begin{array}{l}\mathrm{Co}, \mathrm{Al}-\mathrm{O} 2 \\
\mathrm{Co}, \mathrm{Al}-\mathrm{O} 2^{i} \\
\mathrm{Co}^{\prime} \mathrm{Al}-\mathrm{O} 1^{i} \\
\mathrm{Co}, \mathrm{Al}-\mathrm{O} 3 \\
\mathrm{Co}, \mathrm{Al}-\mathrm{O} 4^{i}\end{array}$ & $\begin{array}{l}1.88(1) \\
1.88(1) \\
1.89(2) \\
1.97(1) \\
2.29(1)\end{array}$ & $\begin{array}{l}i=1-y, 1-x, z \\
i=1+1 / 2-x, 1 / 2-y, 1 / 2+z \\
i=1+x, y, z\end{array}$ \\
\hline $\begin{array}{l}\mathrm{P}-\mathrm{O} 3 \\
\mathrm{P}-\mathrm{O} 2^{i} \\
\mathrm{P}-\mathrm{O} 2^{i} \\
\mathrm{P}-01\end{array}$ & $\begin{array}{l}1.43(1) \\
1.53(1) \\
1.53(1) \\
1.56(2)\end{array}$ & $\begin{array}{l}i=1 / 2+y, 1+1 / 2+x, 1 / 2-z \\
i=-1 / 2+x, 1 / 2-y, 1 / 2-z\end{array}$ \\
\hline $\begin{array}{l}\mathrm{N}-\mathrm{C} \\
\mathrm{C}-\mathrm{C}^{i}\end{array}$ & $\begin{array}{l}1.52(2) \\
1.61(3)\end{array}$ & $i=x,-y, 1-z$ \\
\hline
\end{tabular}

${ }^{a}$ Standard deviations in parentheses. The positions of the atoms, with reference to the positions listed in Table 10, are indicated by the $i$-values. structure along [001]. The oxygen atom $\mathrm{O} 3$ has larger thermal displacement parameters than the other atoms of the framework. This possibly indicates a disordered arrangement of $\mathrm{O} 3$. The ethylenediamine molecule is placed in the channels of the structure with the carbon atoms arranged statistically in the site $16 j$.

\section{Conclusion}

The use of hydrofluoric acid as a mineralizer in the hydrothermal and organothermal synthesis of ZnAPO-5 and CoAPO-5 resulted in single crystals large enough for traditional single crystal X-ray analysis. The templates di- $n$-propylamine, tripropylamine and triethylamine were used. With the template 1,4-diazabicyclo[2.2.2]octane the hydrothermal synthesis gave ZnAPO-35, LEV. The template ethylenediamine gave the zinc-substituted aluminophosphate of the ACP-2 type, and this type of structure was also obtained for a zinc phosphate. The use of hydrofluoric acic is a mineralizer did not result in single crystal of these con. younds.

The organothermal synthesis of the $\mathrm{Co}^{2+}$-substituted aluminophosphates gave with the template ethylenediamine the compounds $\mathrm{CoAl}\left(\mathrm{PO}_{4}\right)_{2} \cdot \mathrm{C}_{2} \mathrm{H}_{9} \mathrm{~N}_{2}$ and the cobalt aluminophosphate of the ACP-3 type. A cobalt phosphate could also be made at the same experimental conditions with the ACP-3 type structure. Using hydrofluoric acid as a mineralizer in the organothermal and the hydrothermal reactions resulted in several cases in the formation of CoAPO-43, GIS. Single crystals were obtained also in the organothermal and hydrothermal syntheses with ethylenediamine as the template in syn- 
theses where the mineralizer hydrofluoric acid was omitted.

Acknowledgements. The Danish Natural Science Research Council has supported this investigation with grants. Carlsbergfondet is acknowledged for financial support by purchase of the Siemens SMART diffractometer. Mrs. C. Secher, Mrs. M. A. Chevallier and Mr. A. Lindahl are thanked for valuable assistance.

\section{References}

1. Wilson, S. T., Lok, B. M. and Flanigen, E. M. U.S. Pat. 4310440 (1982).

2. Flanigen, E. M., Lok, B. M., Patton, R. L. and Wilson, S. T. Stud. Surf. Sci. Catal. 28 (1986) 103.

3. Radaev, S. F., Joswig, W. and Baur, W. H. J. Mater. Chem. 6 (1996) 1413.

4. Kupermann, A., Nadimi, S., Oliver, S., Ozin, G. A., Garcés, J. M. and Olken, M. M. Nature (London) 365 (1993) 239.

5. Huo, Q. and $\mathrm{Xu}, \mathrm{R}$. J. Chem. Soc., Chem. Commun. (1990) 783.

6. Meier, W. M. and Olson, D. H. Atlas of Zeolite Structure Types. Butterworth-Heinemann, London 1992.

7. Altomare, A., Cascarano, G., Giacovazzo, C.,
Guagliardi, A., Burla, M. C., Polidori, G. and Camalli, M. J. Appl. Crystallogr. 27 (1994) 435.

8. Busing, W. R., Martin, K. O. and Levy, H. A. ORFLS, $A$ Fortran Crystallographic Least Squares Program, Report ORNL-TM 305. Oak Ridge National Laboratory, Oak Ridge, TN 1962. LINUS is a 1971 version of ORFLS.

9. Cromer, D. T. and Waber, J. T. Report LA-3056, Los Alamos Scientific Laboratory of the University of California, Los Alamos, NM 1964.

10. Boultif, A. and Louer, D. J. Appl. Crystallogr. 24 (1991) 987.

11. Lok, B. M., Messina, C. A., Patton, R. L., Gajek, R. T., Cannan, T. R. and Flanigen, E. M. J. Am. Chem. Soc. 106 (1984) 6092.

12. Feng, P., Bu, X and Stucky, G. D. Nature (London) 388 (1997) 735.

13. Bu, X., Feng, P., Gier, T. and Stucky, G. D. J. Solid State Chem. 136 (1998) 210.

14. Zabukovec, N., Golic, L., Fajdiga, P. and Kaucic, V. Zeolites 15 (1995) 104.

15. Pluth, J. J., Smith, J. V. and Bennett, J. M. J. Am. Chem. Soc. 111 (1989) 1692

16. Yvon, K., Jeitschko, W. and Parthé, E. J. Appl. Crystallogr. 10 (1977) 73.

17. Chen, J., Jones, R. H., Natarajan, S., Hursthouse, M. B. and Thomas, J. M. Angew. Chem., Int. Ed. Engl. 33 (1994) 639.

Received December 14, 1998. 